\title{
TRANSPORT ANOMALIES AND MARGINAL FERMI-LIQUID EFFECTS AT A QUANTUM CRITICAL POINT
}

\author{
D. BELITZ AND SHARON L. SESSIONS \\ Department of Physics and Materials Science Institute, University of Oregon, Eugene, \\ OR 97403, USA (e-mail: belitz@greatwhite.uoregon.edu) \\ T.R. KIRKPATRICK \\ Insitute for Physical Science and Technology, and Department of Physics, University of \\ Maryland, College Park, MD 20742 \\ M.T. MERCALDO \\ Dipartimento di Fisica "E.R. Caianello" and Istituto Nazionale di Fisica per la Materia, \\ Universitá di Salerno, I-84081 Baronissi (SA), Italy \\ R. NARAYANAN AND THOMAS VOJTA \\ Theoretical Physics, University of Oxford, OX1 3NP, UK
}

\begin{abstract}
The behavior of the conductivity and the density of states, as well as the phase relaxation time, of disordered itinerant electrons across a quantum ferromagnetic transition is discussed. It is shown that critical fluctuations lead to anomalies in the temperature and energy dependence of the conductivity and the tunneling density of states, respectively, that are stronger than the usual weak-localization anomalies in a disordered Fermi liquid. This can be used as an experimental probe of the quantum critical behavior. The energy dependence of the phase relaxation time at criticality is shown to be that of a marginal Fermi liquid.
\end{abstract}

\section{Introduction}

\subsection{Disordered Fermi Liquids}

It is well known that the interplay between quenched disorder and electron-electron interaction effects lead to non-analytic dependencies of various observables on control parameters. For instance, in three-dimensions (which we will assume throughout) the conductivity has a square-root temperature dependence, 1

$$
\sigma(T)=\sigma_{0}[1+\text { const. } \times \sqrt{T}],
$$

the density of states has a square-root energy dependence,

$$
N(\epsilon)=N_{F}[1+\text { const. } \times \sqrt{\epsilon}],
$$

and the phase relaxation rate has an $\epsilon^{3 / 2}$ energy dependence 3

$$
\tau_{\text {ph }}^{-1}(\epsilon)=\text { const. } \times \epsilon^{3 / 2} .
$$

These effects, and similar other ones, are collectively known as 'weak- pcalization effects'. They were originally derived by means of perturbation theory, $\$$ and were later understood to be the leading corrections to scaling at a stable renormalizationgroup fixed point that describes a disordered Fermi liquid 0 They can also be interpreted as an example of 'generic scale invariance', i.e. the occurrence of powerlaw correlations in an entire phase, rather than only at an isolated critical point. 6

manchester: submitted to World Scientific on October 28, 2018 


\subsection{Quantum Critical Behavior of Itinerant Ferromagnets}

Usually, a ferromagnetic phase transition is triggered by changing the temperature through the Curie point. The resulting critical behavior is caused by thermal fluctuations that become large near the critical point, and is referred to as 'thermal' or 'classical' critical behavior. However, a system with a low Curie temperature can also be driven into the paramagnetic phase by means of a non-thermal control parameter, such as pressure, or composition, at zero or very low temperature. An example is $\mathrm{MnSi}$, which undergoes a ferromagnet-to-paramagnet transition at $T=0$ and a hydrostatic pressure $p_{c} \approx 15 \mathrm{kbar} 0$ This is an example of a 'quantum critical point' 8 . The critical behavior is driven by quantum fluctuations, and it is therefore different from that at the corresponding thermal phase transition.

The critical behavior at the quantum ferromagnetic transition of disordered itinerant electrons has recently been determined exactly, 10 and was found to consist of power laws 11 with log-log-normal corrections to scaling. For instance, the magnetization behaves like

$$
m(t) \propto t^{2} e^{(\ln \ln (1 / t))^{2}},
$$

where $t$ is the dimensionless distance from the critical point at $T=0$, e.g., $t=$ $\left|p-p_{c}\right| / p_{c}$. In writing Eq. (何), we have omitted constant prefactors in the exponent.

Equation (4) determines the critical exponent $\beta$, which is defined by the behavior of the magnetization as a function of $t, m(t) \propto t^{\beta}$. A convenient way to account for the fact that, due to the logarithmic corrections to scaling, the asymptotic critical behavior is not simply given by power laws, is to formally make the critical exponents scale dependent. We thus write

$$
\beta=2+\ln g(\ln b) / \ln b \quad,
$$

with $b$ an arbitrary renormalization group length scale factor, and $g$ a function that, for large arguments and omitting constant prefactors, can be represented by $g(x) \approx$ $\exp \left[(\ln x)^{2}\right]$. More generally, one needs three independent critical exponents to describe quantum critical behavior. One of these is the dynamical critical exponent $z$, which determines the scaling behavior of frequency and temperature. It reads 10

$$
z=3+\ln g(\ln b) / \ln b \quad .
$$

In addition to Eq. (6) there is a second time scale in the problem which plays an important role in the complete theory $1 \mathrm{~d}$ but is not crucial for our present purposes.

For the second static exponent we choose the correlation length exponent $\nu, 10$

$$
1 / \nu=1+\ln g(\ln b) / \ln b \quad .
$$

Finally, for later reference, we note that the scale dimension of the leading irrelevant operator in a disordered electron system, which we denote by $u$, is given by -1.0

Notice that the critical behavior is not mean-field like, in contrast to the prediction by Hertz. 3 The physical reason for this breakdown of Hertz's order parameter field theory lies in the existence of soft modes in itinerant ferromagnets, viz. particle-hole excitations, that are distinct from the order parameter fluctuations, but couple sufficiently strongly to the latter to influence the critical behavior.

manchester: submitted to World Scientific on October 28, 2018 


\section{Feedback of critical behavior on weak-localization effects}

The particle-hole excitations that are responsible for the non-mean field critical behavior described above are the same soft modes that lead to the weak-localization nonanalyticities. One should therefore expect the critical behavior to change the latter via feedback effects. This is indeed the case, as has been shown in Ref. 12 .

The easiest way to determine this effect is by means of scaling arguments. Let us start with the electrical conductivity. This transport coefficient is unrelated to magnetism, so at a magnetic phase transition one expects its scale dimension to be zero. We thus have a scaling or homogeneity law

$$
\sigma(t, T)=F_{\sigma}\left(t b^{1 / \nu}, T b^{z}, u b^{-1}\right)
$$

with $F_{\sigma}$ a scaling function. Using the fact that $F_{\sigma}(0,1, x)$ is an analytic function of $x$, and putting $b=T^{-1 / 3}$, we obtain at criticality, $t=0$,

$$
\sigma(t=0, T)=\sigma_{0}\left[1+c_{\sigma}\left[\left(T / T_{F}\right) g\left(\ln \left(T_{F} / T\right)\right)\right]^{1 / 3}+O(\sqrt{T})\right] .
$$

Here $\sigma_{0}$ is the disordered Fermi-liquid value of $\sigma, T_{F}$ is the Fermi temperature, and $c_{\sigma}$ is a disorder dependent constant.

Similarly, the leading correction to the density of states is given by an integral over a four-fermion correlation function 2 . 3 whose diffusive dynamics lead to $\Delta N \sim$ $b^{-1}$. We thus have

$$
\Delta N(t, \epsilon)=b^{-1} F_{N}\left(t b^{-1 / \nu}, \epsilon b^{z}\right),
$$

with $F_{N}$ another scaling function. At criticality this yields

$$
N(t=0, \epsilon)=N_{F}\left[1+c_{N}\left[\left(\epsilon / \epsilon_{F}\right) g\left(\ln \left(\epsilon_{F} / \epsilon\right)\right)\right]^{1 / 3}+O(\sqrt{\epsilon})\right],
$$

with $N_{F}$ the disordered Fermi liquid value of the density of states at the Fermi level, and $\epsilon_{F}$ the Fermi energy.

Finally, in a disordered electron system the phase relaxation rate scales like a wavenumber squared, and thus has a scale dimension of 2 . This implies

$$
\tau_{\mathrm{ph}}^{-1}(t, \epsilon)=b^{-2} F_{\mathrm{ph}}\left(t b^{-1 / \nu}, \epsilon b^{z}, u b^{-1}\right),
$$

with $F_{\mathrm{ph}}$ a third scaling function. The leading irrelevant variable $u$ represents interaction effects that are necessary for any dephasing, and the rate is therefore proportional to $u$. At criticality, and with a constant $c_{\mathrm{ph}}$, this leads to

$$
\tau_{\mathrm{ph}}^{-1}(t=0, \epsilon)=c_{\mathrm{ph}} \epsilon g\left(\ln \left(\epsilon_{F} / \epsilon\right)\right)+O\left(\epsilon^{3 / 2}\right) .
$$

Equations (11) and (13) remain valid if $\epsilon$ is replaced by $T$ (at $\epsilon=0$ ), and Eq. (9) remains valid if $T$ is replaced by the frequency $\Omega$.

\section{Discussion}

As we have made plausible above, and have shown in more detail in Refs. 12, 10, the transport and thermodynamic properties of disordered electrons show remarkable anomalies at a quantum ferromagnetic critical point. The conductivity and

manchester: submitted to World Scientific on October 28, 2018 
the density of states show nonanalytic dependencies on the temperature or frequency/energy that are similar to, but stronger than, the usual weak-localization anomalies. The crossover from the square-root dependence of the latter to the cuberoot of the former should be easily observable experimentally. Such an experiment would provide a check of the theoretically predicted value $z=3$ of the dynamical critical exponent. For both quantities, there are strong multiplicative corrections to scaling, which in an experiment with a limited dynamical range would result in an effective exponent that is different from 3. For the phase relaxation time, we have found an even more drastic effect: Schmid's $\epsilon^{3 / 2}$ (or $T^{3 / 2}$ ) law gets replaced by a linear energy (or temperature) dependence with a multiplicative logarithmic correction. The electron system at the quantum ferromagnetic critical point thus has single-particle properties that are those of a marginal Fermi liquid.13

\section{Acknowledgments}

Part of this work was performed at the Aspen Center for Physics, and supported by the NSF under grant Nos. DMR-98-70597 and DMR-99-75259, by the DFG under grant No. Vo659/3, and by the EPSRC under grant No. GR/M 04426.

\section{References}

1. B.L. Altshuler, A.G. Aronov, and P.A. Lee, Phys. Rev. Lett. 44, 1288 (1980).

2. B.L. Altshuler and A.G. Aronov, Sol. State Commun. 30, 115 (1979).

3. A. Schmid, Z. Phys. B 271, 251 (1974).

4. For reviews, see, P.A. Lee and T.V. Ramakrishnan, Rev. Mod. Phys. 57, 287 (1985); B.L. Altshuler and A.G. Aronov, in Electron-Electron Interactions in Disordered Systems, edited by M. Pollak and A. Efros (North Holland, Amsterdam 1984), p.1.

5. D. Belitz and T.R. Kirkpatrick, Phys. Rev. B 56, 6513 (1997).

6. T.R. Kirkpatrick and D. Belitz, J. Stat. Phys. 87, 1307 (1997).

7. C. Pfleiderer, G.J. McMullan, S.R. Julian, and G.G. Lonzarich, Phys. Rev. B 55, 8330 (1997).

8. J. A. Hertz, Phys. Rev. B 14, 1165 (1976).

9. See, S. Sachdev, Quantum Phase Transitions (Cambridge University Press, Cambridge 1999).

10. D. Belitz, T.R. Kirkpatrick, M.T. Mercaldo, and Sharon L. Sessions, Phys. Rev. B 63, 174427 (2001); Phys. Rev. B 63, 174428 (2001).

11. T.R. Kirkpatrick and D. Belitz, Phys. Rev. B 53, 14364 (1996).

12. D. Belitz, T.R. Kirkpatrick, R. Narayanan, and Thomas Vojta, Phys. Rev. Lett. 85, 4602 (2000).

13. C.M. Varma, P.B. Littlewood, S. Schmitt-Rink, E. Abrahams, and A.E. Ruckenstein, Phys. Rev. Lett. 63, 1996 (1989).

manchester: submitted to World Scientific on October 28, 2018 